\title{
Research on Caching Strategy in Content Centric Network
}

\author{
Linlin Ma ${ }^{1}$, Jianwei Zhang ${ }^{1+}$, Zengyu Cai ${ }^{1}$, Zhihui Shang ${ }^{1}$, Xuhui Wang ${ }^{1}$ \\ ${ }^{1}$ School of Computer and Communication Engineering, Zhengzhou University of Light Industry, Zhengzhou,
} China

\begin{abstract}
Content Centric Network (CCN) is a new kind of network architecture, which carried on routing, caching based on content. CCN fundamentally changed data package structure and addressing mode, from cared host address to focused on content itself. One of its main features is to leverage built in-network caching to improve the transmission efficiency of receiver-driven content retrieval and network resource utilization. CCN caching takes on several new characteristics: transparency, ubiquity, and fine granularity. However, these characteristics have made traditional caching theories, models and optimization techniques unable to be directly and seamlessly ported to $\mathrm{CCN}$ caching. In this paper, new features of $\mathrm{CCN}$ as well as their ensuing challenges are introduced, mechanisms of optimizing the performance of in-network caching are analyzed and compared from various angles. This paper summarizes and analyzes some key problems to be solved and the direction of future research.
\end{abstract}

Keywords: content centric network, in-network caching, transparency, fine granularity.

\section{Introduction}

$\mathrm{CCN}$ is one of the 4 future Internet infrastructure projects, which was supported by the United States NSF in August 2010. CCN changed current Internet host based peer to peer communication architecture, to support efficient scalable communication mode. At present, $\mathrm{CCN}$ research is at the initial stage, it still faces many problems such as routing, caching, transmission. CCN uses in-network caching technology, which not only designed a cache server in edge network, but also demand that each node in the network has the function of routing and caching. Nodes can cache data packets according to the specific routing and caching strategy, making that the cache of covering whole network becomes a part of network architecture. When the same user requests arrive to the node, the node can directly return data without forwarding user request to the content source. In recent years, many researchers have been dedicated to the research of CCN caching, and they have achieved creative research results in many research fields such as theory, model and optimization methods. Based on this background, this paper reviews the current research results of CCN caching. Through compared the related research ideas, this paper points out the future research directions and challenges.

\section{CCN and in-network cache characteristics}

\section{1. $\mathrm{CCN}$ architecture}

CCN separates content and address information, instead of the traditional network idea regard IP address as the center. So users are not care about the contents address, and only concern about the content itself. $\mathrm{CCN}$ has two types of message types: Interest Packet and Data Packet(in Fig 1), Three types of data structures: Content Store (CS), Pending Interest Table (PIT), Forwarding Information Base (FIB). CCN adopts the requests driver model, in turn queries CS, PIT, FIB , to forward Interest packets. The node model of $\mathrm{CCN}$ is shown in Fig 2.

\footnotetext{
+ Corresponding author. Tel.: +13603829696; fax: +037163556921

E-mail address: ing@zzuli.edu.cn.
} 


\begin{tabular}{|c|c|}
\multicolumn{2}{c|}{ Interest packet } \\
\hline Content Name \\
\hline $\begin{array}{c}\text { Selector } \\
\text { order } \\
\text { preference,filter,scope.. }\end{array}$ & Content Name \\
\cline { 2 - 2 } & $\begin{array}{c}\text { Signed Info(publisher ID,key } \\
\text { locator...) }\end{array}$ \\
\hline Nonce & Data \\
\hline
\end{tabular}

Fig. 1: CCN Package Type

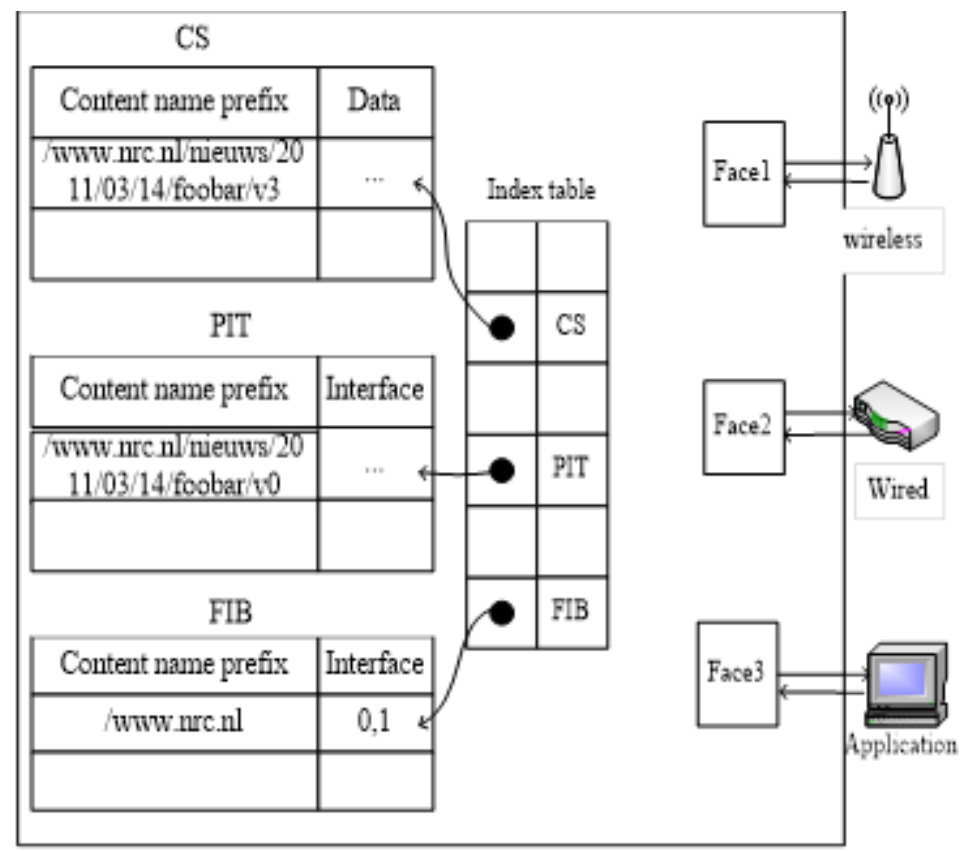

Fig. 2: Forwarding engine model

\subsection{In-network cache characteristics}

\section{(1) cache transparency}

Traditional caching exists usually in the form of a particular closed system for a single business type, such as Web, P2P, CDN. P2P services mostly adopted private protocols, which made each P2P application become a closed system. So it was difficult to achieve the sharing of cache resources. At present, some researchers have conducted research to achieve P2P cache transparency, such as the DECAED working group [1]. Although Web caching system was based on the open HTTP protocol, Web content was based on domain independent naming, the same object can not be uniquely identified, so content object of the cache system was logically isolated from the autonomous domain. The problems mentioned above were mainly derived from the closure of communication protocol, and the inconsistency of naming methods. CCN architecture was proposed to solve the above problem.CCN globally unique identified content, like IP address, to achieve named consistency, while these global content identification can achieve self verification [2]. In network layer, $\mathrm{CCN}$ systems are based on content identifier for content routing and caching decisions, which enabled caching and application separation. These features made caching to be a universal, open, and transparent service.

\section{(2) cache ubiquity}

In traditional cache system, The topology of network cache was generally linear cascade structure or hierarchical tree structure, the cache node positions were usually fixed. In CCN, the cache was widespread, the cache nodes were not fixed, caching topological structure can be used to describe any network structure, the relationship between upstream and downstream nodes was not clear. These increased the difficulty of mathematical modeling and performance analysis of cache system, also improved the inter cache 
coordination difficulty. Meanwhile, high dynamic system has brought great challenge to the consistency of the cache system. Therefore, in a high dynamic environment of in-network caching, how to maintain the availability of objects and optimize object's acquisition cost were some problems to be solved urgently.

(3) cache fine granularity

Traditional contents cache unit was a document or fragment. CCN cache unit was the content chunk of fine grained, it had the global content identification. It went on caching, replacement with content chunk, which met the requirement that caching node operated with line speed[3]. The larger file is divided into relatively small content chunk, and different content block from a file can be obtained from different network nodes. Based on content block for data replacement, it improved efficiency of content retrieval and cache space utilization. However, some cache replacement algorithms based on file size were no longer applicable.

\section{CCN in-Network Cache Research Progress}

In $\mathrm{CCN}$ communications, in order to improve scalable and efficient content distribution, optimizing cache service performance became very important. In figure 3, we shown the existing cache research of CCN, it can be divided into 4 aspects: cache decision strategy, cache resource allocation, cache replacement algorithm, cache mode.

\subsection{Cache decision strategy}

Cache decision strategy refers to what object should cache in which nodes, and select what decision algorithm. Specific content includes that where cache the return contents, what contents need to cache in nodes, how to cache contents in nodes. Based on the complexity of collaboration between nodes, it will generally be divided into explicit collaboration and implicit collaboration decision strategy. In CCN, due to cache nodes were not fixed, cache flow types were diversity, cache operation required line speed, yet explicit collaborative decision algorithm has high computational complexity and high cost of information interaction. Therefore, CCN needs to adopt simple implicit caching decision strategies. The default cache placement strategy of CCN was LCE (Leave Copy Everywhere), namely in the transmission process, all nodes along the way can cache the object copy. In order to reduce the redundant of cache system, on the basis of LCE, researchers have proposed LCD (Leave Copy Down), MCD (Move Copy Down), Prob (Copy with probability) [4]. Recently several new implicit cache collaborative decision strategy are proposed. Literature[5] proposed cooperative caching strategy based on age. When new contents arrive, the node will cache a copy, and according to the distance at the server, content popularity and other factors add a age value in data reported. This scheme made the content stored in the network edge, reduced unnecessary storage and eased the load on server. Cui XD, Liu J et al designed in-network cache strategy based on node number and replacement rate[6]. By weighing the importance of node location and the cache content timeliness to achieve the best position of the return content. This strategy have been achieved lower loading in the source server and less average hops than Betw strategy and ALWAYS strategy. Based on content popularity and center node matching strategy was proposed[7]. Through selective caching content, it improved efficiency of the use of cache space along nodes, and the content diversity of caching system.

\subsection{Cache resource allocation}

The cache space of network nodes directly affect the performance of cache system. Therefore reasonable allocating cache space for each node is very important. Psaras[8] et al proposed that distributed more cache space to edge nodes, which can effectively improve the network performance. Then a kind of Cooperative Caching Mechanism with Content Migration(CCMCM) is proposed[9]. In this scheme, the centrality of node was considered in the selection of caching nodes to ensure that contents can be cached in the more important nodes an much as possible. When the cached contents are extensive, the caching node can transfer some contents to the appropriate neighbor according to the cache space available, the cache replacement rate and the connection stability between nodes. Literature[10] designed a hierarchical CCN network cache placement policy. We build a network CCN hierarchical caching model, through hierarchical configuration node cache size and function space, the popularity of different content can be placed on different layers CCN nodes in different layers. So it improved the resource allocation efficiency of different nodes, while improved the cache performance of the overall optimal cache performance in $\mathrm{CCN}$. 


\subsection{Cache replacement algorithm}

In $\mathrm{CCN}$, typical cache replacement strategy included random replacement algorithm, least frequently used(LFU) algorithm and least recently used(LRU) algorithm[11]. They respectively replaced and updated randomly selected content, used the minimum frequency content for a long time, and the least recently used content. CCN required the cache to be executed at a linear speed, so a relatively simple cache replacement algorithm was needed. Recently, the research shown that the simple random replacement algorithm in CCN can achieve the performance of the LRU replacement algorithm.

\subsection{Cache mode}

$\mathrm{CCN}$ in-network cache mode adopted distributed cache. Distributed cache that information block storage, refers to intermediate network nodes mutual cooperation, joint consultation to store the complete information. In the $\mathrm{CCN}$ environment, each autonomous system is a content provider. They should store the most valuable information on the entire Internet to balance their self-centered behavior.In literature[12], collaborative caching and routing scheme based on local request similarity was proposed. It effectively combined the redundant eliminate on the vertical request path and content placement in horizontal local scope, which reduced the content request delay and cache redundancy, and with a small amount of additional costs for the decline in content request overhead. Literature [13] presented a novel algorithm for cooperative caching based on node similarity. Interest packet was forwarded to the most similar node to increase the hit rate for correlate requests. Curbing the same duplication between collaborative nodes, the algorithm reduces the redundancy while increasing the diversity. In Literature [14], first an energy consumption model for content distribution was built and a judging condition for energy efficiency optimization in caching was designed. On this basis, an Energy-Efficiency Aware Probability Caching(E2APC)scheme was proposed. This scheme can effectively reduce the whole energy consumption, while guaranteeing comparatively high cache hit rate and few average response hops.

\section{Future Research Directions and Challenges}

Although content center network has been widely concerned, and has achieved some results, there are still many problems:

(1) Network business and behavior with statistical characteristics. For uncertain network environment, based on the method of probability statistics, designing related methods and strategies of network will obtain better performance. So it will be a very valuable study that research and design the model of probabilistic cache in caching system.

(2) The size of the cache space of network nodes. Nodes of CCN are operated at line speed, the larger the caching space, the higher the complexity of processing, the greater the network overhead, which limits the size of caching space of nodes. Therefore it has been a problem how large cache space the nodes is allocated can improve the performance of cache system.

(3) Cache decision algorithm and cache replacement algorithm. CCN goes on caching and operating based on the content, which determines the between of request content object has a certain correlation .

(4) Low complexity cooperative caching strategy. In CCN, the caching is universal, cache node is no longer fixed, the network topology has changed from the hierarchical tree structure to the mesh map, the relationship between the upper and lower node also becomes uncertain. These factors increase the difficulty of mathematical modeling and analysis, and enhance the difficulty of inter-cache coordination. Therefore, it needs to design a simplified and efficient caching cooperative mechanism [15-16].

\section{Conclusions}

With the explosive growth of network scale, the diversification development of new business, constantly upgrade of intelligent terminal. The commonly information processing functions need to be added to the network nodes, to achieve content efficient distribution. Therefore, based on transparency, ubiquitous innetwork caching also needs continuous improvement..

This paper analyzes the characteristics of in-network caching. Then, concludes the performance optimization scheme of CCN cache system, including cache decision strategy, cache resource allocation, 
cache replacement strategy, cache mode. Finally, put forward the research direction for further study. At present, the research of CCN cache technology is still in its infancy, and many problems need to be solved and perfected step by step. These have important theoretical value and practical guiding significance for planning, design and operation of the $\mathrm{CCN}$ network in the future.

\section{Acknowledgements}

This paper was supported by National Natural Science Foundation (61672471); 2016 Outstanding Innovation Science and Technology Youth Project of the Innovation Scientists and Technicians Plan of Henan Province(164100510019); Science and Technology Key Project of Zhengzhou Science and Technology Development Plan(153PKJGG26); 2016Zhengzhou University of Light Industry graduate student science and Technology Innovation Fund.

\section{References}

[1] DECADE. HTTPS://datatracker.ietf.org/wg/decade.

[2] Ahlgren B, Dannewitz C, Imbrenda C, et al. A survey of information-centric networking. Communications Magazine, IEEE. 2012,50(7): 26-36.

[3] Xia CM, Xu MW. Summary of information center network research. Computer science and exploration. 2013, 7(6): 481-493.

[4] Zhang GQ, Li Y, Tang H. Survey of in-network caching techniques in information-centric networking. Journal of Software. 2014, 25(1): 154-175

[5] Ming Z, Xu M,Wang D. Age-based cooperative caching in information-centric netwoeks//Proc of conference on computer communication workshops.[s.1.]:IEEE. 2012, pp. 268-273 .

[6] Cui XD, Liu J, Huang T, Chen JY, Liu YJ. A Novel In-network caching scheme based on betweenness and replacement rate in content centric network. Journal of Electronics and Information Technology. 2014, 36(1): 1-7.

[7] Rui LL, Peng H, Huang HQ, Qiu XS, Shi RC. Popularity and centrality based selective caching scheme for information-centric networks. Journal of Electronics and Information Technology. 2016, 38(2): 325-331.

[8] Psaras I, Chai W K, Pavlou G. Probabilistic in-network caching for information-centric networking. In Proceedings of the second edition of the ICN workshop on Information-centric networking. 2012, pp. 55-60.

[9] Luo X, An Y, Yu JX, Liu Y. Cooperative caching mechanism with content migration in content-centric network. Journal of Electronics and Information Technology. 2015, 37(11): 2790-2794.

[10] Xia L, Wang L, Zhang CC, Lv W. Hierarchical caching mechanism study for content centric network. Microelectronics \& Computer. 2016, 33(2): 22-26.

[11] Jelenkovic P R, Radovanovic A, Squillante MS. Critical sizing of lru caches with dependent requests. Journal of Applied Probability. 2006, pp. 1013-1027.

[12] Ge GD, Guo YF, Liu CX, Lan JL. Collaborative caching and routing scheme based on local request similarity in named data networking. Journal of Electronics \& Information Technology. 2015, 37(2): 435-442.

[13] Fang XW, Chen ZQ, Ren ZR, Jang YM. Collaborative caching algorithm based on node similarity in content centric networking. Computer Science. 2016, 43(4): 81-86.

[14] Luo X, An Y, Yu JX, Liu Y. Energy-efficiency aware probabilistic caching scheme for content centric network. Journal of Electronics \& Information Technology. 2016, 38(8): 1843-1849.

[15] Hu Q, Wu MQ, Guo S, Peng L. Random cache placement strategy for content centric network. Journal of XIDIAN Universal. 2014, 41(6): 131-136.

[16] Ge GD, Guo YF, Liu CX, Lan JL. A collaborative caching strategy for privacy protection in content centric network. Journal of Electronics \& Information Technology. 2015, 37(5): 1220-1226. 